\title{
Research on Watermarking Technology of Printing Image in CMYK Space Based on Dual-Tree Complex Wavelet
}

\author{
Shen Lijun ${ }^{1}$, Wang Wenju ${ }^{1+}$, Cui Ruibo ${ }^{1}$, Jiang Zhongmin ${ }^{1}$, Li Jinming ${ }^{1}$, Tang Lijing ${ }^{1}$, Sun \\ Liujie $^{1}$ \\ ${ }^{1}$ Shanghai University of Science and Technology, Shanghai 200093, China
}

\begin{abstract}
In the process of watermark embedding and extraction, there is a great contradiction between the invisibility and robustness of the watermark. In order to solve this contradiction, this paper presents a semiblind watermarking algorithm for printing image anti-counterfeiting in CMYK space, based on Arnold scrambling, 2D-DCT and dual-tree complex wavelet transform (DT-CWT). First, Arnold Scrambling is applied to the watermark image. Then the scrambled watermark image is transformed by 2D-DCT to obtain the frequency domain image. Second, the obtained frequency domain image is used to replace the sub-band containing the least information in the wavelet coefficient tree obtained from DT-CWT of the carrier CMYK image, and then applying invers dual-tree complex wavelet transform to the decomposed wavelet coefficient tree to get the watermarked CMYK image. The extraction process uses module matching technology to perform optimal watermark extraction on the watermarked images. Experimental results show that the algorithm not only has good confidentiality, but also can resist various attacks such as cropping, rotation, filtering, noise addition, lossy compression, etc. It is very suitable for anti-counterfeiting authentication of printed products.
\end{abstract}

Keywords: DT-CWT, arnold scrambling, 2D-DCT, module matching.

\section{Introduction}

Now, how to protect the intellectual property of printed works has become an urgent problem to be solved. The principle of the Anti-counterfeit technology is adding digital watermark information to the carrier image before outputting the printed image [1].

As we all know, printing machines use the CMYK color space for color rendering. In order to reduce the loss of information and improve the robustness of the algorithm, this paper chooses to embed watermark information in the CMYK color space. [2]

Watermark embedding techniques are commonly classified into two categories, i.e. spatial and transform domain based techniques [3] [4], because frequency domain technology has a strong ability to resist attacks, now the frequency domain watermarking algorithm occupies the mainstream. In 2017, Summuyya Munib and Asifullah Khan [5] proposed a watermarking algorithm based on robust image triangle region and Zernike matrix. Their idea is as follows: according to the image feature points and the local Zernike matrix to embed the watermark information; The Zernike matrix for each selected triangle segment is calculated and then use the dithering modulation to embed the watermark into the Zernike matrix. However, the anti-attack capability of this technology is very poor, and the extraction process is complex. Last but not least, the calculation amountto of it is large and the effect of it is not good.

In this paper, the author uses the characteristics of Arnold Scrambling, 2D-DCT and DT-CWT, combined with the mature wavelet tree theory and Human Visual System (HSV) characteristics to propose a

\footnotetext{
${ }^{+}$Corresponding author:. Tel: 13636675266 ;
}

E-mail address: wangwenju666@163.com 
semi-blind watermark algorithm for the copyright protection of printed images [6]. This algorithm has high efficiency and can well resolve the contradiction between the imperceptibility and robustness of the watermark. It has strong invisibility, high robustness.

\section{Basic Knowledge}

\subsection{DT-CWT}

DT-CWT [7] is a new wavelet transform proposed by Nick Kingsbury et al. and it aims to improve the DWT with poor translation sensitivity and weak direction selectivity. DT-CWT uses two DWT transformations of the binary tree structure, one tree generates the transformed real part, and the other tree generates the transformed imaginary part. The process is shown in the Figure 1.

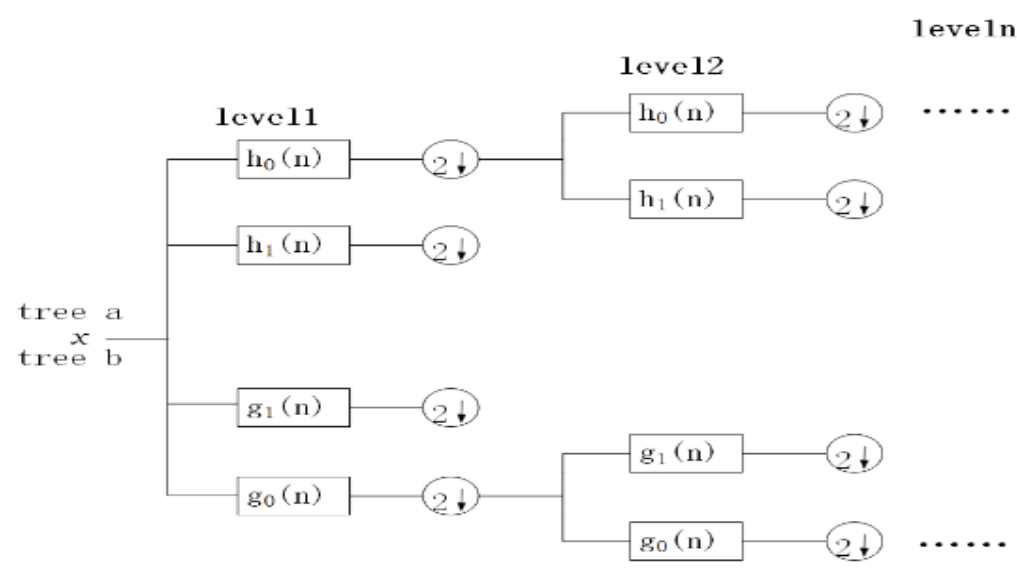

Fig. 1: DT-CWT decomposition diagram

(Note:h0 (n) 、h1 (n) 、g0 (n) 、g1 (n) are low-pass/high-pass filters of tree a and tree b. “2 $\downarrow$ ” Indicates the process of double sampling.)

1D-DT-CWT is expressed as formula (1):

$$
\psi(\mathrm{t})=\psi_{\mathrm{h}}(\mathrm{t})+\mathrm{j} \psi_{g}(t)
$$

In the formula (1), $\psi(\mathrm{t})$ is a complex wavelet, $\psi_{\mathrm{h}}(\mathrm{t})$ is a wavelet of tree a, and $\psi_{g}(t)$ is a wavelet of tree $b$.

Let $H\left(\ell^{j \omega}\right)$ and $G\left(\ell^{j \omega}\right)$ be discrete Fourier transforms of $\mathrm{h}(\mathrm{n})$ and $\mathrm{g}(\mathrm{n})$ respectively, then the lowpass/high-pass filter bank needs to satisfy the following conditions:

(1) perfect reconstruction.

$$
H_{0}\left(\ell^{j \omega}\right) \tilde{H}_{0}\left(\ell^{j \omega}\right)+H_{1}\left(\ell^{j \omega}\right) \tilde{H}_{1}\left(\ell^{j \omega}\right)=2
$$

(2) $\psi_{g}(t)$ is the approximate Hilbert transform of $\psi(\mathrm{t}) . \mathrm{h}_{0}(\mathrm{n})$ and $\mathrm{g}_{0}(\mathrm{n})$ should satisfy formula (3).

$$
g_{0}(0) \approx h_{0}(n-0.5)
$$

For two-dimensional images, 2D-DT-CWT is needed, that is DT-CWT should perform on the rows and columns of the image respectively.

\section{Research on Dual-tree Complex Wavelet Watermarking Technique in CMYK Color Space}

\subsection{Watermark embedding}




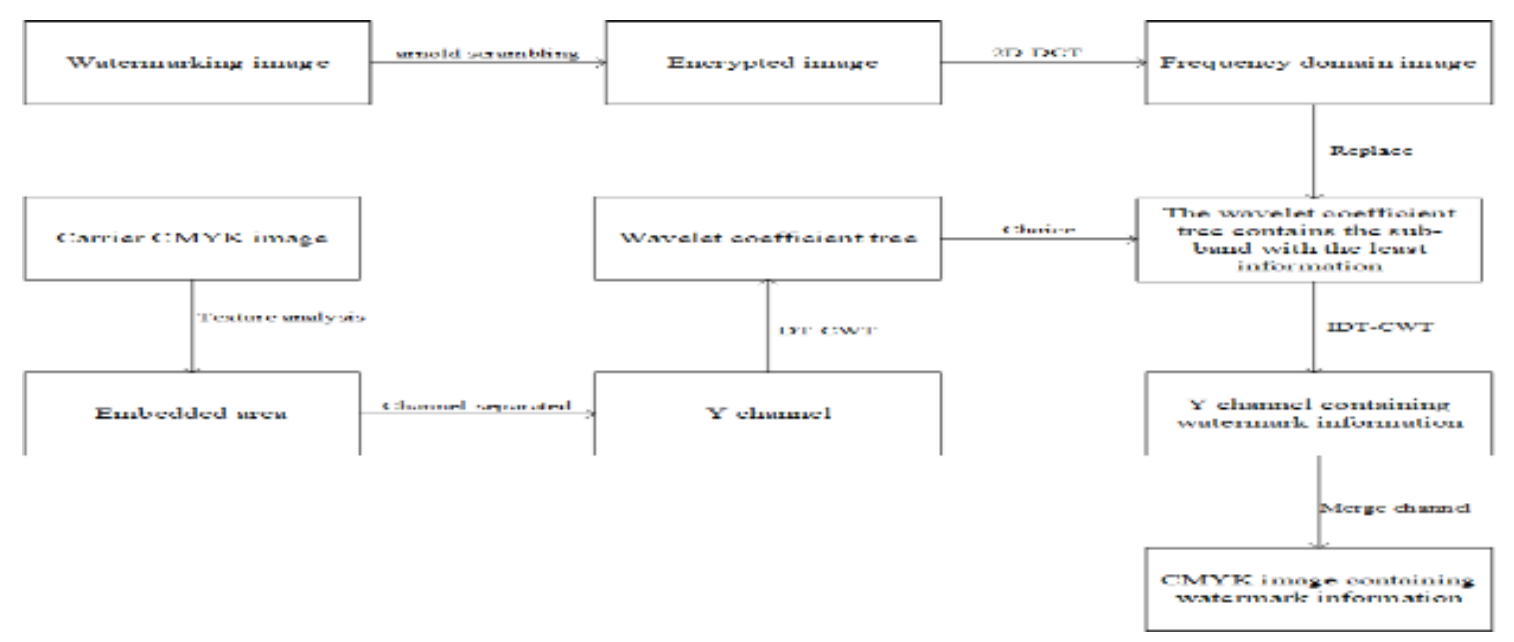

Fig. 2: Watermark embedding flow chart

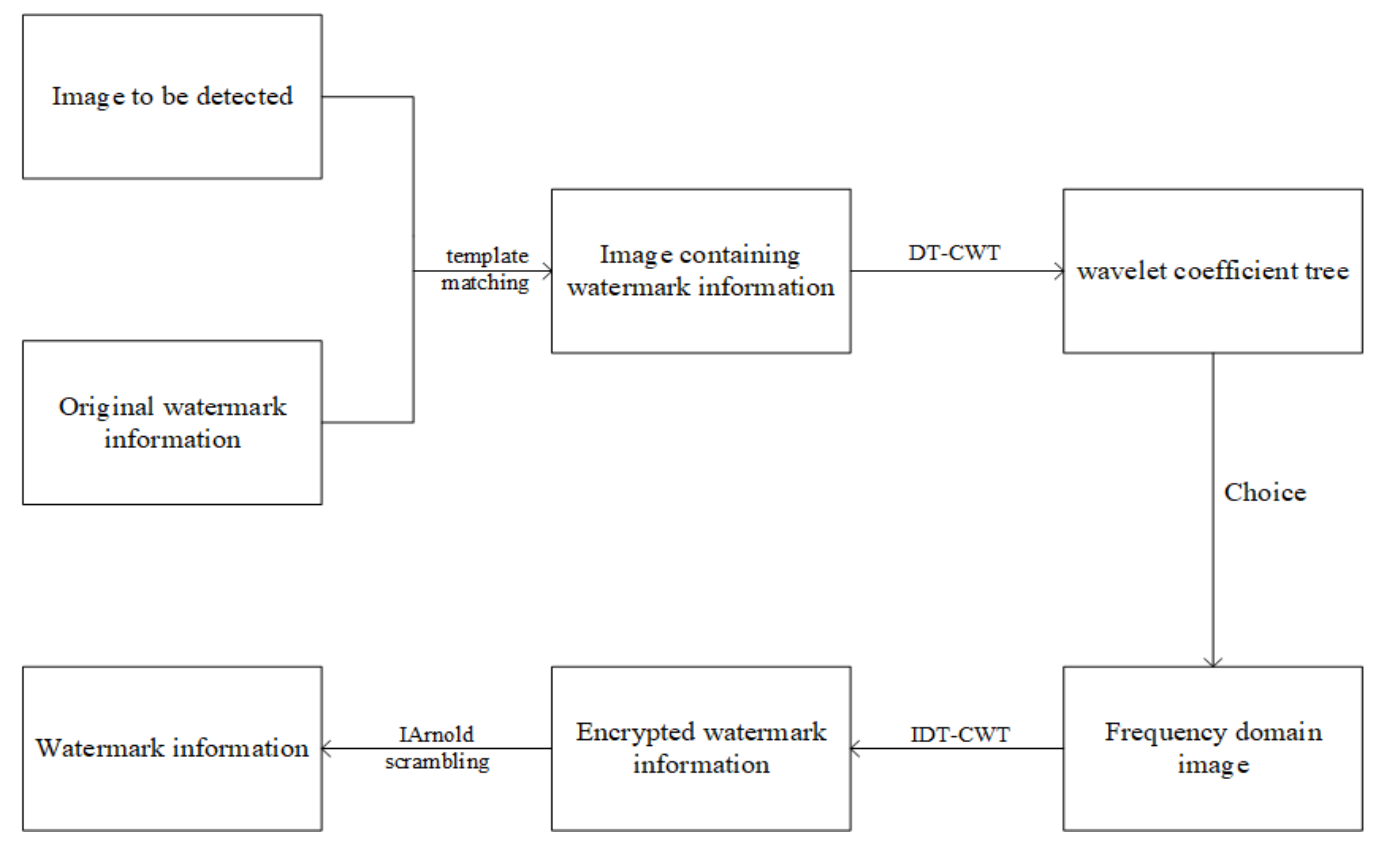

Fig. 3: Watermark extraction flow chart

1) Firstly, Arnold scrambling should be performed on the binary watermark image for 20 times to generate an encrypted image, and then 2D-DCT is performed on the encrypted image to obtain a frequency domain image.

2) Secondly, the carrier CMYK image is read , and then an area should be selected to embed the watermark.

The width and height of this area both take 4 times as long as the watermark image. The position of the selected region is characterized by the feature texture analysis of the co-occurrence matrix of the carrier CMYK image [8]. The author selects the area where the texture groove is deep and the effect is clear. Contrast (CON) directly reflects the comparison of the brightness of a certain pixel value and the pixel value in its domain, and the clarity of the image and the degree of texture groove depth. In theory, if the contrast is small, the groove pattern is shallow and the effect is blurred. CON is calculated by formula (4).

$$
C O N=\sum_{i} \sum_{j}(i-j)^{2} P(i, j)
$$

3) Thirdly, the channels of the selected region image should be separated, and then 2-level DT-CWT is done to the $\mathrm{Y}$ channel image. There are $6 \mathrm{~J}$ detail sub-bands and 2 approximation sub-bands after the decomposition. The $\mathrm{J}$ is a positive integer, and the selection range of the $\mathrm{J}$ value is determined by formula (5):

$$
J \leq \min \left(\left\lfloor\log _{2} M\right\rfloor,\left\lfloor\log _{2} N\right\rfloor\right)
$$


The values of $\mathrm{M}$ and $\mathrm{N}$ in equation (9) are the width and height of the selected area. \lfloor\rfloor$_{\text {indicates the }}$ downward rounding. Through many experiments, the author gets the best result when $\mathrm{J}=2$.

4) Fourthly, the author select the appropriate replacement strength $K$ and uses the frequency domain image to replace the sub-band with the least information contained in the wavelet coefficient tree. Two inverse wavelet transforms are performed on all sub-bands to obtain a Y channel image containing watermark information. The $\mathrm{Y}$ channel image containing the watermark information is merged with the $\mathrm{C}, \mathrm{M}, \mathrm{K}$, channel images of the carrier CMYK image to get the watermarked CMYK image. The watermark embedding process is shown in Figure 2.

\section{Watermark extraction}
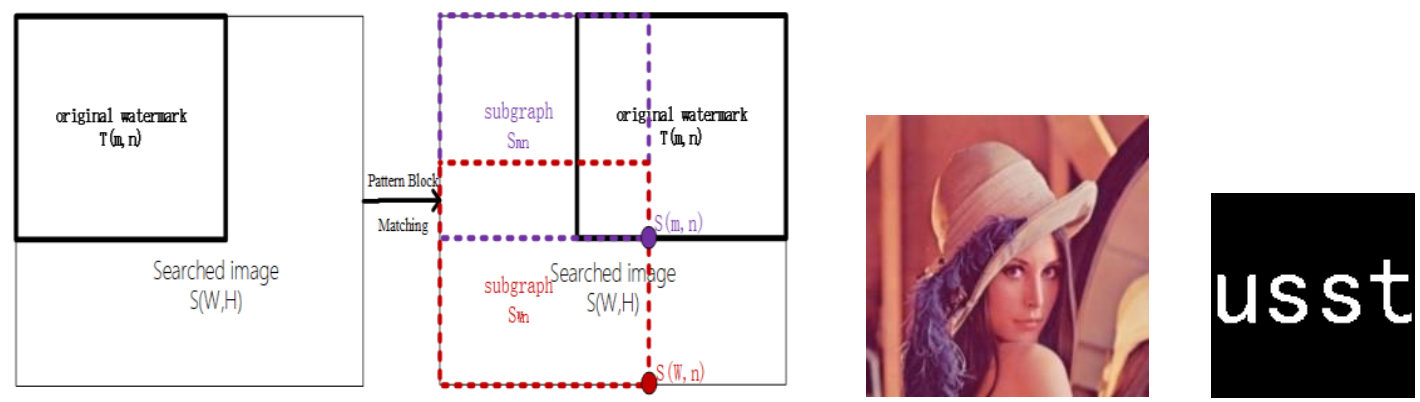

Fig. 4: Module matching and search process diagram

Fig. 5a: Carrier image

Fig. 5b: Watermarking image

1) The image to be detected should be scanned in the mode of CMYK and be matched with the original watermark image ${ }^{[9]}$. The matching process is shown in Figure4.

2) The DT-CWT is performed on the proposed sub-block containing watermark information to obtain the transformed wavelet coefficient tree.

3) Perform 2D-DCT on the subband that has been replaced in the process of embedding watermark. Then the encrypted watermark information is obtained.

4) The encrypted watermark information is subjected to the IAranold Scrambling to obtain the original watermark information. Because the entire process of reconstructing the watermark does not require the participation of the original carrier image, it belongs to a semi-blind extraction technique. Watermark extraction process is shown in Figure 2.

\section{Experiments and Results}

\subsection{Watermark invisibility experiments and results}

MATLAB simulation software is used to test the watermark invisibility and the quality of the extracted watermark. As shown in Figure 5a, the carrier image is a CMYK image of 512 pixels by 512 pixels. Figure $5 \mathrm{~b}$, the watermark image is a binary image with a sign USST and the size of the image is 127 pixels by 127 pixels.

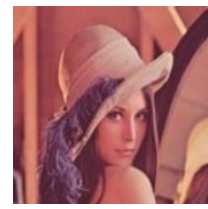

Fig. 6a

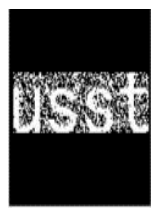

Fig. 6b

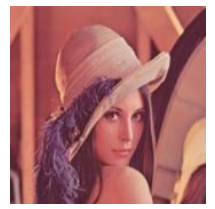

Fig. 7a

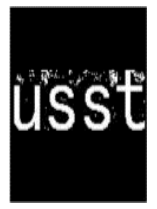

Fig. 7b

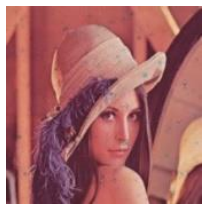

Fig. 8a

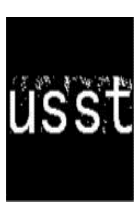

Fig. 8b

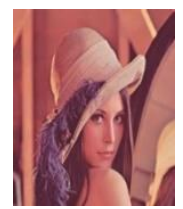

Fig. 9a Fig. 9b

PSNR (Peak Signal-to-Noise Ratio) is an important index for evaluating image's quality. The PSNR is used to evaluate the quality of image (invisibility of the watermark).

NC (normalized correlation coefficient) is used to measure the degree of similarity between the extracted watermark and the original watermark (the quality of the reconstructed watermark). The similarity between the reconstructed watermark and the original watermark are evaluated objectively by using the NC value.

Table 1 is used to describing the NC and PSNR of Figure 6a to Figure 9b: 
Table 1: Results of invisible experiment

\begin{tabular}{ccccc}
\hline $\begin{array}{c}\text { Replacement method } \\
\text { (Replacement strength })\end{array}$ & DWT (50) & $\begin{array}{c}\text { DT-CWT } \\
(50)\end{array}$ & DWT (800) & DT-CWT (800) \\
\hline PSNR & 50.154 & 42.245 & 17.215 & 38.546 \\
NC & 0.6792 & 0.8544 & 0.9123 & 0.9831 \\
\hline
\end{tabular}

By observing, it can be intuitively found that both Figure6a and Figure7a have strong invisibility, but Figure6b is obviously more obscure than Figure 7b; Both Figure8b and Figure $9 b$ are clear, but there are many visible spots on Figure8a; By comparing the data in Table 1 (the lager the PSNR value, the closer the image is to the original image; the closer the $\mathrm{NC}$ is to 1 the clearer the reconstructed watermark). It is accurately draw that compared with the most people commonly use DWT to embed the watermark, the author's method is better. The method proposed in this paper improves the invisibility of the watermark, making it possible to embed the watermark strongly without destroying the quality of the original image. In addition, the quality of the extracted watermark is also significantly higher than that of the watermark, which is usually extracted by the DWT method.

\subsection{Robust experiments and results}

Printer and Scanner are used to test the robustness of the watermark algorithm. The replacement intensity of the image used in the experiment was 150, and the replacement channel was the Y channel.

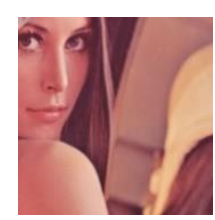

Fig. 10a

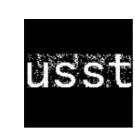

Fig. 10b

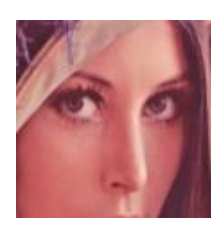

Fig. 11a

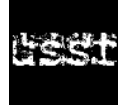

Fig. 11b

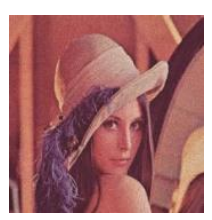

Fig. 14a

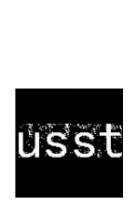

Fig. $14 \mathrm{~b}$

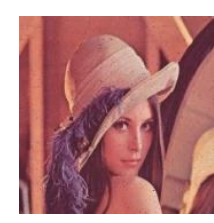

Fig. 15a

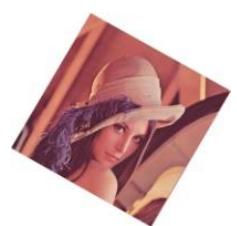

Fig. 12a

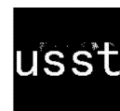

Fig. 12b

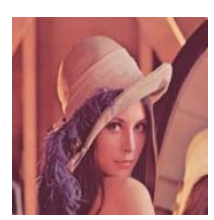

Fig. 13a

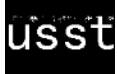

Fig. 13b

\subsubsection{Anti-cut test:}

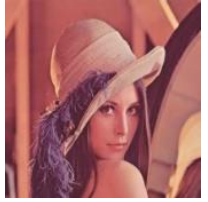

Fig. 16a

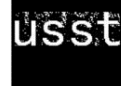

Fig. 16b

Figure10a is the remaining part of the watermarked image whose $75 \%$ is removed. Figure $10 \mathrm{~b}$ is extracted from Figure10a, and its NC is 0.8993; Figure11a is the remaining part of the watermarked image whose $91.84 \%$ is removed. Figure 11b shows the watermark extracted from Figure 11a. The watermark's NC is 0.7111 . Experimental results fully demonstrate that this algorithm has strong anti-cutting ability.

\subsubsection{Anti-rotation experiment:}

Figure $12 \mathrm{a}$ is obtained by rotating the watermarked image for $30^{\circ}$. Figure $12 \mathrm{~b}$ is extracted from Figure 12a, and its NC is 0.9688 . Experimental result indicate that this algorithm has good anti-rotation ability.

\subsubsection{Filter experiment And Noise-added experiment:}

Figure13a is obtained by performing median filtering of the watermarked image with using the template of $3 * 3$. Figure $13 \mathrm{~b}$ is extracted from Figure $13 \mathrm{a}$, and its NC is 0.9028 ; Figure 14a is obtained by performing 0.02 Gaussian noise enhancement on the watermarked image. Figure14b is extracted from Figure 14a, and its $\mathrm{NC}$ is 0.9170 ; Figure $15 \mathrm{a}$ is obtained by adding 0.01 pepper salt noise to the watermarked image. Figure $15 \mathrm{~b}$ is extracted from Figure 15a, and its NC is 0.9076. The experimental results indicate that this algorithm has strong anti-noise and filtering capabilities.

\subsubsection{Compression experiment:}


Figure 16a is obtained by compressing the watermarked image with JPEG by $54 \%$. Figure $16 \mathrm{~b}$ is extracted from Figure 16a and its $\mathrm{NC}$ is 0.9088 . The experimental results show that: that the algorithm has strong anticompression ability;

\section{Conclusion}

In the process of analysing Arnold scrambling and 2D-DCT, the author found that the frequency domain image generated by combining the two features of Arnold scrambling and 2D-DCT is very smooth, and the encrypted watermark information is evenly distributed on the frequency domain image. Then the dual-tree complex wavelet is used to embed the watermark, which makes the reconstructed watermark clearer. The image is processed in the CMYK color space, which avoids the loss of watermark information that caused by the color gamut conversion. By observing the picture, it is verified that the watermark embedded image also satisfies human visual perception compared with the original carrier image, which is shown that the algorithm has good invisibility. By analysing the experimental results of anti-cutting, anti-rotation, anti-noise addition, and anti-compression, it is shown that the algorithm has good robustness. In the process of watermark extraction, the participation of the original image information is not required, so this is a semiblind watermark algorithm.

\section{Acknowledgements}

This work was supported in part by a grant from Laboratory of Green Platemaking and Standardization for Flexography Printing (ZBKT201710), the Innovation and Entrepreneurship Project of College Student of University of Shanghai for Science and Technology (No.XJ2017238, XJ10252292, XJ10252296, XJ10252297) and the Innovation and Entrepreneurship Project of College Student of Shanghai (SH10252168, SH017163).

\section{References}

[1] Thongkor, Kharittha. Amornraksa, Thumrongrat. Digital image watermarking for printed and scanned documents. Source: Proceedings of SPIE - The International Society for Optical Engineering. 2017. v 10420.

[2] Wang, CaiYin. Kong, XiangWei. Li, Chao. Robust Watermarking Applying to CMYK Printed Image for Fidelity Printing. Source: Dianzi Keji Daxue Xuebao/Journal of the University of Electronic Science and Technology of China. March 1, 2017. v 46, n 3, p 529-536.

[3] Kumar, Sanjay. Dutta, Ambar. Performance analysis of spatial domain digital watermarking techniques. Source: 2016 International Conference on Information Communication and Embedded Systems, ICICES 2016. July 21.

[4] Pardhu, Thottempudi. Perli, Bhaskara Rao. Source: Digital image watermarking in frequency domain International Conference on Communication and Signal Processing, ICCSP 2016. 2016. p 208-211.

[5] Munib, Summuyya. Khan, Asifullah. Robust image watermarking technique using triangular regions and Zernike moments for quantization based embedding. Source: Multimedia Tools and Applications. March 1, 2017. v 76, n 6, p 8695-8710.

[6] Singh, Siddharth. Rathore, Vivek Singh. Singh, Rajiv, Manoj Kumar Singh. semi-blind image watermarking in redundant wavelet domain Source: Multimedia Tools and Applications. September 1, 2017. v 76, n 18, p 1911319137.

[7] Selesnick, Ivan W. Baraniuk, Richard G. Kingsbury, Nick G. The dual-tree complex wavelet transform Source: IEEE Signal Processing Magazine. November 2005. v 22, n 6, p 123-151.

[8] Kiss, Csaba Istvan. Nemeth, Geza. Chetverikov, Dmitrij. Boroczky, Lilla. On feature extraction for texture analysis. Source: Periodica Polytechnica Electrical Engineering. 1995. v 39, n 2, p 115-130.

[9] Wong, Ze - Hao. Abdullah, K. Wong, C.J. Template matching using multiple templates weighted normalised cross correlation. Source: ISCAIE 2014 - 2014 IEEE Symposium on Computer Applications and Industrial Electronics. January 14, 2015. p 131-135. 